\title{
Binary Representation of Natural Numbers
}

\author{
Hiroyuki Okazaki $\left.\right|^{1}$ \\ Shinshu University \\ Nagano, Japan
}

\begin{abstract}
Summary. Binary representation of integers [5, 3, and arithmetic operations on them have already been introduced in Mizar Mathematical Library 8. 7, 6, 4. However, these articles formalize the notion of integers as mapped into a certain length tuple of boolean values.

In this article we formalize, by means of Mizar system [2, [1, the binary representation of natural numbers which maps $\mathbb{N}$ into bitstreams.
\end{abstract}

MSC: 68W01 68T99 03B35

Keywords: algorithms

MML identifier: BINARI_6, version: 8.1.08 5.53.1335

\section{Preliminaries}

Let us consider a natural number $x$. Now we state the propositions:

(1) There exists a natural number $m$ such that $x<2^{m}$.

(2) If $x \neq 0$, then there exists a natural number $n$ such that $2^{n} \leqslant x<2^{n+1}$. Proof: Define $\mathcal{Q}\left[\right.$ natural number] $\equiv x<2^{\$_{1}}$. There exists a natural number $m$ such that $\mathcal{Q}[m]$. Consider $k$ being a natural number such that $\mathcal{Q}[k]$ and for every natural number $n$ such that $\mathcal{Q}[n]$ holds $k \leqslant n$. Reconsider $k_{1}=k-1$ as a natural number. $2^{k_{1}} \leqslant x$.

(3) Let us consider a natural number $x$, and natural numbers $n_{1}, n_{2}$. If $2^{n_{1}} \leqslant x<2^{n_{1}+1}$ and $2^{n_{2}} \leqslant x<2^{n_{2}+1}$, then $n_{1}=n_{2}$.

${ }^{1}$ This study was supported in part by JSPS KAKENHI Grant Numbers JP17K00182. The author would also like to express gratitude to Prof. Yasunari Shidama for his support and encouragement. 
(4) $\langle 0\rangle=\langle\underbrace{0, \ldots, 0}_{1}\rangle$.

(5) Let us consider natural numbers $n_{1}, n_{2}$. Then $\langle\underbrace{0, \ldots, 0}_{n_{1}}\rangle \sim\langle\underbrace{0, \ldots, 0}_{n_{2}}\rangle=$ $\langle\underbrace{0, \ldots, 0}_{n_{1}+n_{2}}\rangle$.

2. Homomorphism from the Natural Numbers to the Bitstreams

Let $x$ be a natural number. The functor LenBinSeq $(x)$ yielding a non zero natural number is defined by

(Def. 1) (i) it $=1$, if $x=0$,

(ii) there exists a natural number $n$ such that $2^{n} \leqslant x<2^{n+1}$ and it $=$ $n+1$, otherwise.

Let us consider a natural number $x$. Now we state the propositions:

(6) $x<2^{\operatorname{LenBinSeq}(x)}$.

(7) $x=\operatorname{AbsVal}(\operatorname{LenBinSeq}(x)$-BinarySequence $(x))$. The theorem is a consequence of $(6)$.

(8) Let us consider a natural number $n$, and an $(n+1)$-tuple $x$ of Boolean. If $x(n+1)=1$, then $2^{n} \leqslant \operatorname{AbsVal}(x)<2^{n+1}$.

(9) There exists a function $F$ from Boolean* into $\mathbb{N}$ such that for every element $x$ of Boolean*, there exists a (len $x$ )-tuple $x_{0}$ of Boolean such that $x=x_{0}$ and $F(x)=\operatorname{Abs} \operatorname{Val}\left(x_{0}\right)$.

Proof: Define $\mathcal{P}$ [element of Boolean ${ }^{*}$, object] $\equiv$ there exists a (len $\left.\$_{1}\right)$ tuple $x_{0}$ of Boolean such that $\$_{1}=x_{0}$ and $\$_{2}=\operatorname{AbsVal}\left(x_{0}\right)$. For every element $x$ of Boolean ${ }^{*}$, there exists an element $y$ of $\mathbb{N}$ such that $\mathcal{P}[x, y]$. Consider $f$ being a function from Boolean* into $\mathbb{N}$ such that for every element $x$ of Boolean $* \mathcal{P}[x, f(x)]$.

The functor Nat2BinLen yielding a function from $\mathbb{N}$ into Boolean* is defined by

(Def. 2) for every element $x$ of $\mathbb{N}$, it $(x)=\operatorname{LenBinSeq}(x)$-BinarySequence $(x)$.

Now we state the propositions:

(10) Let us consider an element $x$ of $\mathbb{N}$, and a (LenBinSeq $(x))$-tuple $y$ of Boolean. If $(\operatorname{Nat} 2 \operatorname{BinLen})(x)=y$, then $\operatorname{AbsVal}(y)=x$. The theorem is a consequence of (7).

(11) $\operatorname{rng}$ Nat2BinLen $=\left\{x\right.$, where $x$ is an element of Boolean ${ }^{*}: x($ len $x)=$ $1\} \cup\{\langle 0\rangle\}$. 
Proof: For every object $z, z \in \operatorname{rng}$ Nat2BinLen iff $z \in\{x$, where $x$ is an element of Boolean $\left.{ }^{*}: x(\operatorname{len} x)=1\right\} \cup\{\langle 0\rangle\}$.

(12) Nat2BinLen is one-to-one.

Let $x, y$ be elements of Boolean*. Assume len $x \neq 0$ and len $y \neq 0$. The functor MaxLen $(x, y)$ yielding a non zero natural number is defined by the term (Def. 3) $\max (\operatorname{len} x$, len $y)$.

Let $K$ be a natural number and $x$ be an element of Boolean*. The functor $\operatorname{ExtBit}(x, K)$ yielding a $K$-tuple of Boolean is defined by the term

(Def. 4) $\begin{cases}x \frown\langle\underbrace{0, \ldots, 0}_{K-^{\prime} \operatorname{len} x}\rangle, & \text { if len } x \leqslant K, \\ x\lceil K, & \text { otherwise. }\end{cases}$

Now we state the propositions:

(13) Let us consider a natural number $K$, and an element $x$ of Boolean*. Suppose len $x \leqslant K$. Then $\operatorname{ExtBit}(x, K+1)=\operatorname{ExtBit}(x, K)^{\frown}\langle 0\rangle$.

(14) Let us consider a non zero natural number $K$, and an element $x$ of Boolean ${ }^{*}$. If len $x=K$, then $\operatorname{ExtBit}(x, K)=x$.

(15) Let us consider a non zero natural number $K, K$-tuples $x, y$ of Boolean, and $(K+1)$-tuples $x_{1}, y_{1}$ of Boolean. Suppose $x_{1}=x^{\frown\langle 0\rangle}$ and $y_{1}=y^{\frown}\langle 0\rangle$. Then $x_{1}$ and $y_{1}$ are summable.

(16) Let us consider a non zero natural number $K$, and a $K$-tuple $y$ of Boolean. Suppose $y=\langle\underbrace{0, \ldots, 0}_{K}\rangle$. Let us consider a non zero natural number $n$. If $n \leqslant K$, then $y_{/ n}=0$.

(17) Let us consider a non zero natural number $K$, and $K$-tuples $x, y$ of Boolean. Then carry $(x, y)=\operatorname{carry}(y, x)$.

(18) Let us consider a non zero natural number $K$, and $K$-tuples $x, y$ of Boolean. Suppose $y=\langle\underbrace{0, \ldots, 0}_{K}\rangle$. Let us consider a non zero natural number $n$. Suppose $n \leqslant K$. Then

(i) $(\operatorname{carry}(x, y))_{/ n}=0$, and

(ii) $(\operatorname{carry}(y, x))_{/ n}=0$.

Proof: Define $\mathcal{P}$ [natural number] $\equiv$ if $1 \leqslant \$_{1} \leqslant K$, then $(\operatorname{carry}(x, y)) / \$_{1}=$ 0 . For every non zero natural number $i$ such that $\mathcal{P}[i]$ holds $\mathcal{P}[i+1]$. For every non zero natural number $k, \mathcal{P}[k]$.

Let us consider a non zero natural number $K$ and $K$-tuples $x, y$ of Boolean. Now we state the propositions:

(19) $x+y=y+x$. The theorem is a consequence of (17). 
(20) If $y=\langle\underbrace{0, \ldots, 0}_{K}\rangle$, then $x+y=x$ and $y+x=x$.

Proof: For every natural number $i$ such that $i \in \operatorname{Seg} K$ holds $(x+y)(i)=$ $x(i)$.

(21) Let us consider a non zero natural number $K$, and $K$-tuples $x, y$ of Boolean. If $x(\operatorname{len} x)=1$ and $y(\operatorname{len} y)=1$, then $x$ and $y$ are not summable.

Let us consider a non zero natural number $K$ and $K$-tuples $x, y$ of Boolean. Now we state the propositions:

(22) If $x$ and $y$ are summable, then $y$ and $x$ are summable. The theorem is a consequence of (17).

(23) If $x$ and $y$ are summable and $(x(\operatorname{len} x)=1$ or $y(\operatorname{len} y)=1)$, then $(x+$ $y)(\operatorname{len}(x+y))=1$. The theorem is a consequence of (19) and (22).

(24) Let us consider a non zero natural number $K, K$-tuples $x, y$ of Boolean, and $(K+1)$-tuples $x_{1}, y_{1}$ of Boolean. Suppose $x$ and $y$ are not summable and $x_{1}=x^{\frown\langle 0\rangle}$ and $y_{1}=y^{\frown}\langle 0\rangle$. Then $\left(x_{1}+y_{1}\right)\left(\operatorname{len}\left(x_{1}+y_{1}\right)\right)=1$.

Proof: Set $K_{1}=K+1$. Reconsider $S=\operatorname{carry}(x, y)^{\frown}\langle 1\rangle$ as a $K_{1}$-tuple of Boolean. $S_{/ 1}=$ false. For every natural number $i$ such that $1 \leqslant i<K_{1}$ holds $S_{/ i+1}=\left(x_{1 / i} \wedge y_{1 / i} \vee x_{1 / i} \wedge S_{/ i}\right) \vee y_{1 / i} \wedge S_{/ i}$.

Let $x, y$ be elements of Boolean* ${ }^{*}$ The functor $x+y$ yielding an element of

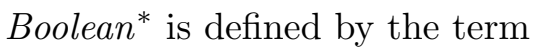

(Def. 5)

$$
\left\{\begin{array}{l}
y, \text { if len } x=0, \\
x, \text { if len } y=0, \\
\operatorname{ExtBit}(x, \operatorname{MaxLen}(x, y))+\operatorname{ExtBit}(y, \operatorname{MaxLen}(x, y)), \\
\quad \text { if } \operatorname{ExtBit}(x, \operatorname{MaxLen}(x, y)) \text { and } \operatorname{ExtBit}(y, \operatorname{MaxLen}(x, y)) \\
\quad \operatorname{are~summable~and~len~} x \neq 0 \text { and } \operatorname{len} y \neq 0, \\
\operatorname{ExtBit}(x, \operatorname{MaxLen}(x, y)+1)+\operatorname{ExtBit}(y, \operatorname{MaxLen}(x, y)+1), \\
\quad \text { otherwise. }
\end{array}\right.
$$

Let $F$ be a function from $\mathbb{N}$ into Boolean ${ }^{*}$ and $x$ be an element of $\mathbb{N}$. Let us note that the functor $F(x)$ yields an element of Boolean ${ }^{*}$. Now we state the propositions:

(25) Let us consider an element $x$ of Boolean*. If $x \in \operatorname{rng}$ Nat2BinLen, then $1 \leqslant \operatorname{len} x$.

(26) Let us consider elements $x, y$ of Boolean*. Suppose $x, y \in \operatorname{rng}$ Nat2BinLen. Then $x+y \in \operatorname{rng}$ Nat2BinLen. The theorem is a consequence of (11), (25), (4), (18), (16), (20), (14), (21), (23), (13), and (24).

(27) Let us consider a non zero natural number $n$, an $n$-tuple $x$ of Boolean, natural numbers $m, l$, and an $l$-tuple $y$ of Boolean. Suppose $y=x^{-}$ $\langle\underbrace{0, \ldots, 0}_{m}\rangle$. Then $\operatorname{AbsVal}(y)=\operatorname{AbsVal}(x)$. 
Proof: Define $\mathcal{P}$ [natural number] $\equiv$ for every natural number $l$ for every $l$-tuple $y$ of Boolean such that $y=x \frown\langle\underbrace{0, \ldots, 0}_{\$_{1}}\rangle \operatorname{holds} \operatorname{AbsVal}(y)=$ $\operatorname{Abs} \operatorname{Val}(x)$. For every natural number $m$ such that $\mathcal{P}[m]$ holds $\mathcal{P}[m+1]$. $\mathcal{P}[0]$. For every natural number $m, \mathcal{P}[m]$.

(28) Let us consider a natural number $n$, an element $x$ of $\mathbb{N}$, and an $n$-tuple $y$ of Boolean. Suppose $y=($ Nat2BinLen $)(x)$. Then

(i) $n=\operatorname{LenBinSeq}(x)$, and

(ii) $\operatorname{AbsVal}(y)=x$, and

(iii) $(\operatorname{Nat} 2 \operatorname{BinLen})(\operatorname{AbsVal}(y))=y$.

The theorem is a consequence of (6).

(29) Let us consider elements $x, y$ of $\mathbb{N}$. Then (Nat2BinLen $)(x+y)=$ $($ Nat2BinLen $)(x)+($ Nat2BinLen $)(y)$. The theorem is a consequence of $(7)$, (27), (26), (28), (13), and (15).

(30) Let us consider elements $x, y$ of Boolean*. If $x, y \in \operatorname{rng}$ Nat2BinLen, then $x+y=y+x$. The theorem is a consequence of (29).

(31) Let us consider elements $x, y, z$ of Boolean*. If $x, y, z \in \operatorname{rng}$ Nat2BinLen, then $(x+y)+z=x+(y+z)$. The theorem is a consequence of (29).

\section{Homomorphism from the Bitstreams to the Natural Numbers}

Let $x$ be an element of Boolean*. The functor $\operatorname{ExtAbsVal}(x)$ yielding a natural number is defined by

(Def. 6) there exists a natural number $n$ and there exists an $n$-tuple $y$ of Boolean such that $y=x$ and $i t=\operatorname{AbsVal}(y)$.

Now we state the proposition:

(32) There exists a function $F$ from Boolean* into $\mathbb{N}$ such that for every element $x$ of Boolean ${ }^{*}, F(x)=\operatorname{ExtAbsVal}(x)$.

Proof: Define $\mathcal{P}$ [element of Boolean ${ }^{*}$, object $] \equiv \$_{2}=\operatorname{ExtAbsVal}\left(\$_{1}\right)$. For every element $x$ of Boolean*, there exists an element $y$ of $\mathbb{N}$ such that $\mathcal{P}[x, y]$. Consider $f$ being a function from Boolean* into $\mathbb{N}$ such that for every element $x$ of Boolean*, $\mathcal{P}[x, f(x)]$.

The functor BinLen2Nat yielding a function from Boolean* into $\mathbb{N}$ is defined by

(Def. 7) for every element $x$ of Boolean ${ }^{*}$, it $(x)=\operatorname{ExtAbsVal}(x)$. 
Let $F$ be a function from Boolean* into $\mathbb{N}$ and $x$ be an element of Boolean* Let us observe that the functor $F(x)$ yields an element of $\mathbb{N}$. Observe that BinLen2Nat is onto.

Now we state the propositions:

(33) Let us consider an element $x$ of Boolean ${ }^{*}$, and a natural number $K$. Suppose len $x \neq 0$ and len $x \leqslant K$. Then $\operatorname{ExtAbsVal}(x)=\operatorname{AbsVal}(\operatorname{ExtBit}(x, K))$. The theorem is a consequence of (27).

(34) Let us consider elements $x, y$ of Boolean*. Then (BinLen2Nat) $(x+y)=$ $($ BinLen2Nat $)(x)+(\operatorname{BinLen2Nat})(y)$. The theorem is a consequence of (33), (13), and (15).

The functor EqBinLen2Nat yielding an equivalence relation of Boolean* is defined by

(Def. 8) for every objects $x, y,\langle x, y\rangle \in$ it iff $x, y \in$ Boolean* $^{*}$ and (BinLen2Nat) $(x)$ $=(\operatorname{BinLen} 2 \mathrm{Nat})(y)$.

The functor QuBinLen2Nat yielding a function from Classes EqBinLen2Nat into $\mathbb{N}$ is defined by

(Def. 9) for every element $A$ of Classes EqBinLen2Nat, there exists an object $x$ such that $x \in A$ and $i t(A)=(\operatorname{BinLen2Nat})(x)$.

Let us observe that QuBinLen2Nat is one-to-one and onto.

Now we state the proposition:

(35) Let us consider an element $x$ of Boolean*. Then $(\mathrm{QuBinLen} 2 \mathrm{Nat})\left([x]_{\text {EqBinLen2Nat }}\right)=($ BinLen2Nat $)(x)$.

Let $A, B$ be elements of Classes EqBinLen2Nat. The functor $A+B$ yielding an element of Classes EqBinLen2Nat is defined by

(Def. 10) there exist elements $x, y$ of Boolean* such that $x \in A$ and $y \in B$ and it $=[x+y]_{\text {EqBinLen2Nat }}$.

Now we state the proposition:

(36) Let us consider elements $A, B$ of Classes EqBinLen2Nat, and elements $x, y$ of Boolean* ${ }^{*}$. If $x \in A$ and $y \in B$, then $A+B=[x+y]_{\text {EqBinLen2Nat }}$. The theorem is a consequence of (34).

Let us consider elements $A, B$ of Classes EqBinLen2Nat. Now we state the propositions:

(37) $($ QuBinLen2Nat $)(A+B)=($ QuBinLen2Nat $)(A)+($ QuBinLen2Nat $)(B)$. The theorem is a consequence of (36), (35), and (34).

(38) $A+B=B+A$. The theorem is a consequence of (36), (35), and (34).

(39) Let us consider elements $A, B, C$ of Classes EqBinLen2Nat. Then $(A+$ $B)+C=A+(B+C)$. The theorem is a consequence of (36), (35), and (34). 
(40) Let us consider a natural number $n$, and elements $z, z_{1}$ of Boolean*. Suppose $z=\varepsilon_{\text {Boolean }}$ and $z_{1}=\langle\underbrace{0, \ldots, 0}_{n}\rangle$.

Then $[z]_{\text {EqBinLen2Nat }}=\left[z_{1}\right]_{\text {EqBinLen2Nat }}$.

(41) Let us consider elements $A, Z$ of Classes EqBinLen2Nat, a natural number $n$, and an element $z$ of Boolean*. Suppose $Z=[z]_{\text {EqBinLen2Nat }}$ and $z=\langle\underbrace{0, \ldots, 0}_{n}\rangle$. Then

(i) $A+Z=A$, and

(ii) $Z+A=A$.

The theorem is a consequence of (40), (36), and (38).

\section{REFERENCES}

[1] Grzegorz Bancerek, Czesław Byliński, Adam Grabowski, Artur Korniłowicz, Roman Matuszewski, Adam Naumowicz, Karol Pąk, and Josef Urban. Mizar: State-of-the-art and beyond. In Manfred Kerber, Jacques Carette, Cezary Kaliszyk, Florian Rabe, and Volker Sorge, editors, Intelligent Computer Mathematics, volume 9150 of Lecture Notes in Computer Science, pages 261-279. Springer International Publishing, 2015. ISBN 978-3319-20614-1. doi 10.1007/978-3-319-20615-8_17.

[2] Grzegorz Bancerek, Czesław Byliński, Adam Grabowski, Artur Korniłowicz, Roman Matuszewski, Adam Naumowicz, and Karol Pakk. The role of the Mizar Mathematical Library for interactive proof development in Mizar. Journal of Automated Reasoning, 61(1):9-32, 2018. do1:10.1007/s10817-017-9440-6

[3] Donald E. Knuth. The Art of Computer Programming, Volume 1: Fundamental Algorithms, Third Edition. Addison-Wesley, 1997.

[4] Hisayoshi Kunimune and Yatsuka Nakamura. A representation of integers by binary arithmetics and addition of integers. Formalized Mathematıcs, 11(2):175-178, 2003.

[5] Gottfried Wilhelm Leibniz. Explication de l'Arithmétique Binaire, volume 7. C. Gerhardt, Die Mathematische Schriften edition, 223 pages, 1879.

[6] Robert Milewski. Binary arithmetics. Binary sequences Formalized Mathematics, 7(1): 23-26, 1998.

[7] Yasuho Mizuhara and Takaya Nishiyama. Binary arithmetics, addition and subtraction of integers. Formalized Mathematics, 5(1):27-29, 1996.

[8] Takaya Nishiyama and Yasuho Mizuhara. Binary arithmetics Formalized Mathematics, 4 (1):83-86, 1993. 